\title{
Development of energy management strategies for the sizing of a fast charging station for electric buses
}

\author{
Iñaki Ojer, Alberto Berrueta, Julio Pascual, Pablo Sanchis, Alfredo Ursúa \\ Department of Electrical, Electronic and Communication Engineering \\ Institute of Smart Cities \\ Public University of Navarre (UPNA) \\ Pamplona, Spain \\ inaki.ojer@unavarra.es
}

\begin{abstract}
The electric vehicle contributes to reduce greenhouse gases emissions and get a greater integration of renewable energy. In this context, the implementation of urban buses implies the development of new grid infrastructures in order to carry out the electricity supply required by the charging stations. In order to optimize the size of these infrastructures and to have more power capacity, this contribution proposes the design and the sizing of a fast charging station with a stationary energy storage system (ESS) installed, to supply the energy of the buses that run on one of the lines of Pamplona (Spain). In this contribution, first, the power demanded by the charging point is measured at the fast charging station located at the Public University of Navarre. Second, three energy management strategies are developed with which to use ESS. Finally, the proposed energy management strategies are simulated with the measured data obtained. The results achieved in this contribution show that with a $40 \mathrm{kWh}$ ESS, the power demanded from the grid can be reduced by a whole $70 \%$, that is, from $250 \mathrm{~kW}$ to $74 \mathrm{~kW}$. In addition, the power contracted in the electricity rate is reduced too.
\end{abstract}

Keywords-fast charging station, electric vehicles, energy management strategies, li-ion battery

\section{INTRODUCTION}

Electric mobility is now a reality, in fact there are several countries that have set a date to ban the sale of internal combustion engine vehicles (ICEV) [1]. For instance, Norway will ban the sale of combustion cars in 2025, the Netherlands and Germany in 2030, and France and Great Britain in 2040. Also, cities such as Barcelona, Paris, London, Rome and Los Angeles will ban the circulation of ICEV through its streets in 2030 , and its bus fleet will be electric in 2025 . The actions of governments, together with the awareness of society, are called to play a role in the spreading of the electric vehicle (EV). A good instance is the case of Norway, where $48 \%$ of the total cars sold in the first quarter of 2019 were electric [2].

The main advantages that EVs offer to the citizens and driver, compared to traditional ICEVs are improved air quality in cities, reduction of noise pollution, lower energy consumption, toll exemption from payment on motorways, free parking, and access to the bus lane in urban areas. The market share of EVs is expected to continue growing, and the overcoming of EVs in service over ICEVs is predicted to take place in 2040 [3].

However, there are three fundamental factors that are limiting the spreading of EVs. The first is related to its reduced autonomy compared to ICEV [4]. However, batteries with increasing energy densities are being developed, which

We would like to acknowledge the support of the European Union under the H2020 project STARDUST (774094), the Spanish State Research Agency (AEI) and FEDER-UE under grants DPI2016-80641-R, DPI201680642-R, PID2019-111262RB-I00 and PID2019-110956RB-I00, the Government of Navarra through research project 0011-1411-2018-000029 GERA and the Public University of Navarre under project ReBMS PJUPNA1904. enlarges the autonomy of EVs. Since 1991, when Sony began to trade Li-ion cells, the specific energy of the batteries has increased by 3.2 times, that is, from $80 \mathrm{Wh} / \mathrm{kg}$ to $256 \mathrm{Wh} / \mathrm{kg}$ [5]. The second factor is the higher cost of an EV compared to a similar ICEV [6]. Nowadays, the investment required to acquire an EV is greater than that for an ICEV with similar characteristics. However, during the next decade the electric car is expected to be cheaper than an ICEV. Some studies estimate that it will be at the end of the decade when this occurs [7], while others predict it by 2022 [3]. The higher cost of electric cars is due to the price of batteries. A few years ago they accounted for half of the total cost of the car, while currently it is estimated to be a third, and in the upcoming years it will only be $25 \%$ of the cost [3]. The last aspect that affects the implementation of the EV, is the scarcity of charging stations. The charging of an EV is a critical factor, given that the energy required by the vehicle is obtained here. In this concern, electrical power is a relevant magnitude since it is directly related to the time required to recharge the batteries. In this sense, slow charging is carried out in times greater than 5 hours, while fast charging takes only a few minutes. In the upcoming years the number of charging stations will increase considerably. Actually, the number of cargo units in Europe is expected to be in 2025 six times larger than in 2018 [8].

Although electric cars have acquired a major role in the electrification of transport, it is electric buses that have currently the greatest impact on the reduction of fusil fuel demand, due to the higher operation hours per vehicle. For instance, 1,000 electric cars relegate about 15 barrels of oil per day. By contrast, the same number of electric buses prevent the consumption of 500 barrels of oil per day [9]. Moreover, the impact of electric buses directly affects the air quality in cities, where these buses operate. In addition, city buses have predictable routes and schedules, making it easier properly install charging points [10]. These are some of the reasons why the electrification of buses will predominate over the rest of the vehicles in the upcoming years [3].

There is a wide range of options to charge electric buses. Among these, the fast charging points are becoming increasingly relevant as they charge the vehicle during the required stop times to pick up and drop off passengers along their routes [10]. The installation of fast charging points entails, a priori, the availability of large power capacity and a large deployment of infrastructure (connections, transformation centers, etc.). However, in order to reduce the power demanded to the grid, some studies and demonstration projects are already being carried out. In this sense, the installation of batteries close to the charging stations has already been studied [11]. In fact, the oil company Shell, which has entered in the electric car charging sector, will install batteries provided by Alfen to minimize the impact of this process on the grid [12]. 
In this contribution, the reduction in the grid connection power of an electric bus charging station is analyzed by adding a stationary energy storage system (ESS) next to the charging point. Various energy management strategies are proposed in order to modify the power demand from the grid. The results obtained by these strategies are analyzed and compared in order to choose the most convenient one.

There are various energy management strategies proposed in the literature. On the one hand, some of them are based on the controlling of the charging station DC bus voltage to manage the battery and photovoltaic generation [13]. On the other hand, the so-called moving average strategies minimize the fluctuations and reduce the power peaks produced by home PV systems [14], [15]. In this contribution a modification of a moving average strategy is proposed in order to adapt it the studied case.

The remaining of this contribution is structured as follows: Section 2 describes the configuration of the Electric Vehicle Smart Charging Station (EV-SCS). In Section 3 the measurements made at the charging point and their analysis are collected. The strategies are detailed in Section 4. Section 5 presents the results obtained from the simulations of the strategies and, finally, the conclusions of the contribution are compiled in Section 6.

\section{DESCRIPTION OF THE CHARGING STATION}

Pamplona, the capital city of Navarre located in the north of Spain, has been promoting sustainable mobility for several years. In this sense, the local mass transit system operator has integrated biogas and hybrid buses into the fleet. Likewise, since March 2019, one of the routes is operated by $100 \%$ electric buses, being this a pioneering initiative in Spain.

A total of six buses continuously run a $12.3 \mathrm{~km}$ distance, divided into a go and return trip. Depending on the day, the bus service and schedule changes. On working days, the six buses are on route, with a trip departing time every 12 minutes. Meanwhile, on Saturdays and holidays, the number of buses in service is reduced to five and three respectively. These electric buses store the required energy in a $44.3 \mathrm{kWh}$ lithium titanate (LTO) battery, with $650 \mathrm{~V}$ of nominal voltage and 550 $\mathrm{kg}$. The route has two fast charging stations located at both extremes of the itinerary, one of them on the campus of the Public University of Navarre. These two stations provide the required energy for each go and return trip, each of them with a length of around $6.5 \mathrm{~km}$. The energy consumption per trip is around $10 \mathrm{kWh}$, and the duration of the charge is 3.5 minutes. Factors such as traffic, weather conditions, number of passengers and driving mode, can modify this value. Fig. 1 shows one of the six electric buses being charged on the campus of the Public University of Navarre. The station includes a pantograph that set the physical connection to the bus through four terminals (positive, negative, ground, and control and communications).

Fig. 2 shows the electrical diagram of the EV-SCS analyzed in this contribution. The station consists of a $300 \mathrm{~kW}$ power supply, fed by a $350 \mathrm{kVA}$ transformation center. The peak power demanded by a regular charge is up to $250 \mathrm{~kW}$. In this context, we study herein the integration of an ESS with the mission of reduce the design power of the grid and the transformer, as well as the contracted power, thus achieving significant savings in the installation and operation of the fast charging station. Likewise, this mode of operation reduces the

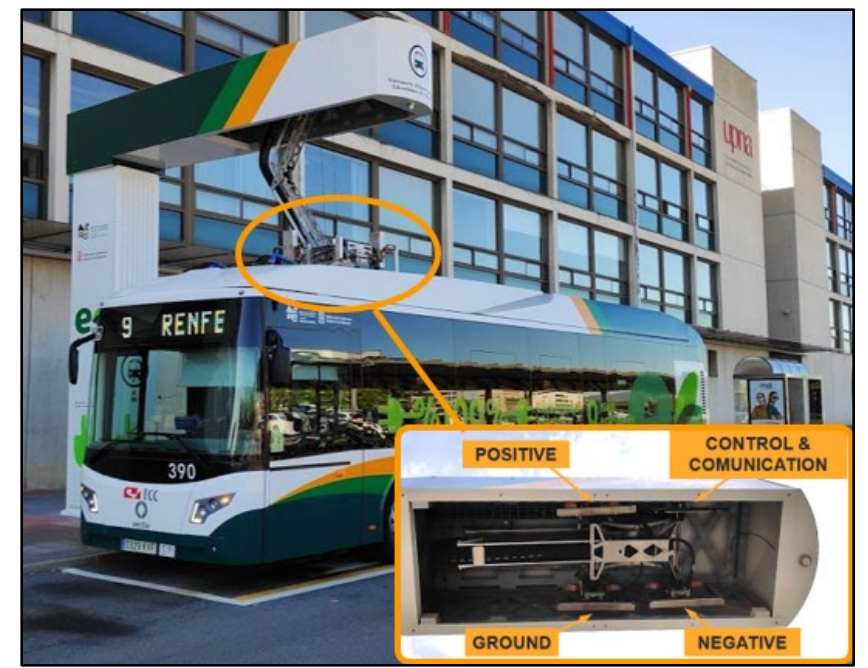

Fig. 1. Electric bus charging station located at Public University of Navarre.

impact of the power peaks demanded from the grid during frequent recharging periods.

The operation mode of the EV-SCS is based on reducing the demand of the grid power $\left(P_{\text {grid }}\right)$ through a power supply from the ESS $\left(P_{E S S}\right)$ to help providing the total charge power required at the charging point $\left(P_{C P}\right)$. Accordingly, approximately the $30 \%$ of the operating time, the buses are connected to the EV-SCS. On the other hand, when the EVSCS is not providing charge to the buses, that is, around $70 \%$ of the operating time, it takes advantage to charge the ESS. This operation mode is based on the following power balance:

$$
P_{\text {grid }}-P_{C P}-P_{E S S}=0
$$

\section{EXPERIMENTAL ANALYSIS OF THE POWER DEMAND TO THE EV-SCS}

In order to study and analyze the power demand at the charging station located at the Public University of Navarre, a Precision Power Analyzer WT3000 has been installed in order to measure the charge power at the charging point for three weeks over three months (April-June 2019).

Comparing the duration of the charges, the anticipation with which they occur with respect to the expected time, the average power and the energy demanded by the buses, great variety among these measurements is reported. It is interesting to know the energy required by the charges, the batteries of the buses demand the energy necessary to be able to make the journey. Also, the duration and the moment in which the charges are made are important because these three parameters will determine the energy consumption of the charging point.

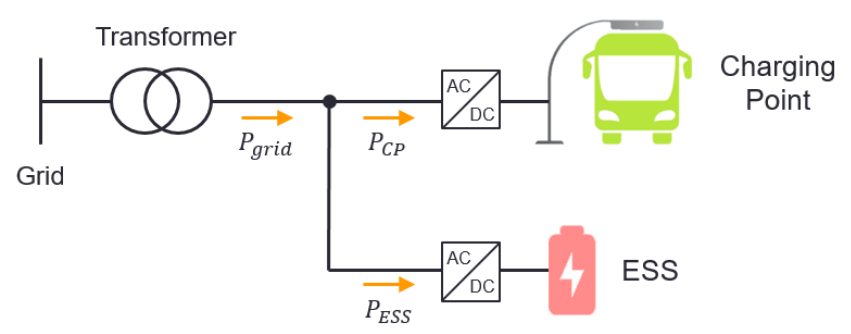

Fig. 2. Electrical diagram of the electric vehicle smart charging station (EVSCS). 
Fig. 3 shows some of the representative charges measured at the charging station. The parameter that is represented is $P_{C P}$. Fig. 3 a) shows a common charge with typical characteristics. It is clearly seen that the charge has two parts, the first gone with increasing power and the second gone with decreasing power. In the first part, the DC current is set at the maximum allowed by the battery and the power increases as the bus battery voltage increases. In the second part, the charge voltage remains constant and the current decreases, therefore the power has a downward slope. This is a very typical charge of lithium-ion batteries, and is known by the acronym CC-CV (constant current - constant voltage) [16]. Fig. 3 b) shows a shorter charge duration. The shorter duration is due a delay in the coming of the bus, which did not provide enough time to carry out the full charge of the battery. In this case the charge becomes so short that there is not even a CV section. At the end of the charge, the state of charge of the battery is lower than that achieved during a typical charge. Fig. 3 c) shows a charge in which the bus has demanded higher energy than during a typical charge, due to a previous deep discharge of the battery.

Fig. 4 represents the average charge power against its duration. The duration and power determine the energy demanded by the bus. This is represented by means of isoenergetic lines added to the graphs. The average value of all charges is highlighted in red, both the point determined by the average power and duration, and the isoenergetic line of the average energy value of all charges.

Fig. 4 clearly shows the great variety of charges at the charging point. On the one hand, there are charges that last less than 1 minute, even some of a few seconds, in which the bus does not even charge $5 \mathrm{kWh}$. On the other hand, there are charges that can last up to 9 minutes, in which the energy demanded is three times higher than expected. Despite this, most charges last between 2 and 5 minutes.

In summary, all the information that has been obtained from the measurement: number of charges made and the percentage of charges made compared to the expected, duration of the charge, the anticipation respect to what was expected, average power of the charge and the energy demanded are collected in Table I, the average values of the three weeks and the total. There are no great differences between the results obtained from the measurements of each

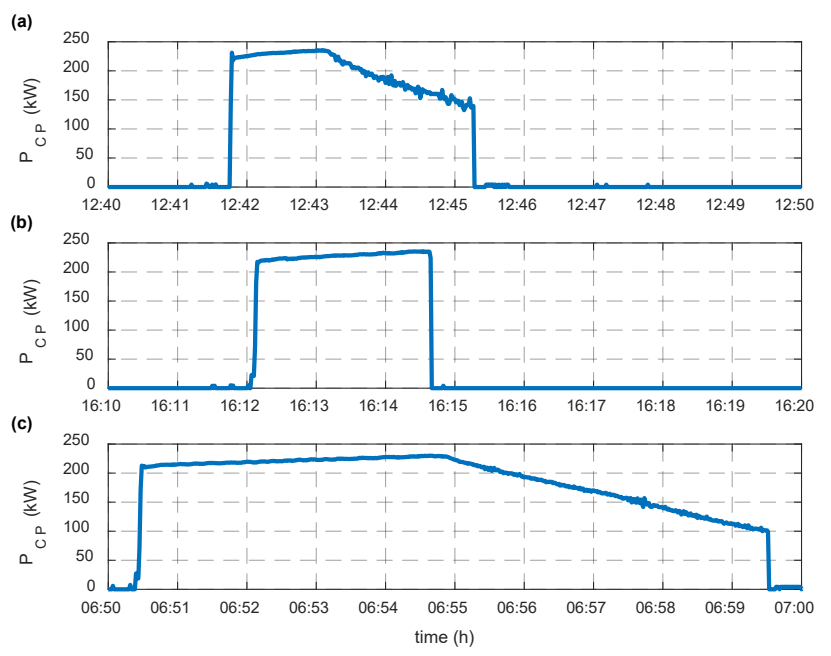

Fig. 3. Examples of some charges measured on April 3, 2019: typical charge (a), short charge (b) and long charge (c). (a)

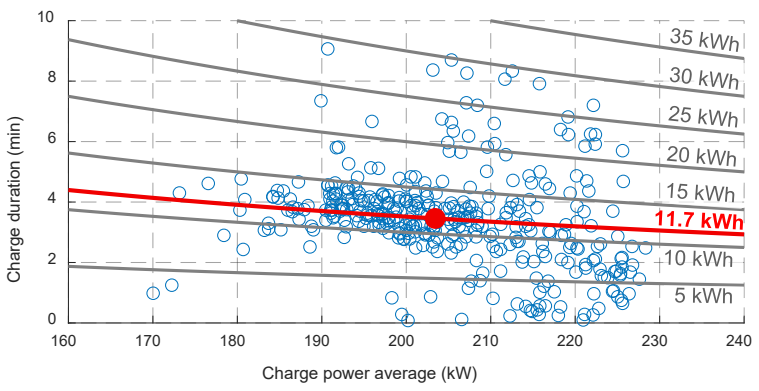

(b)

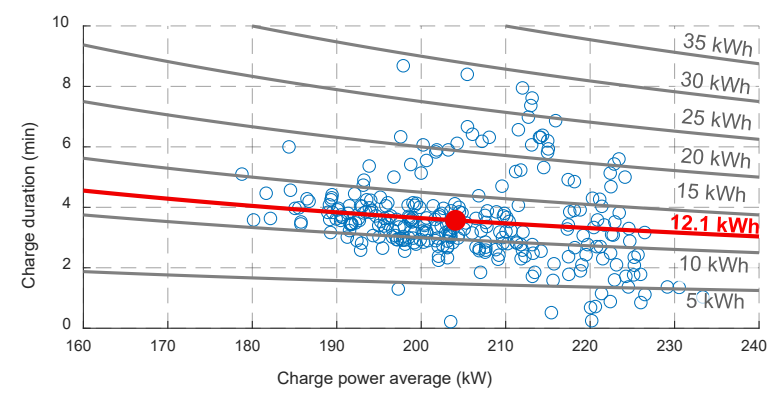

(c)

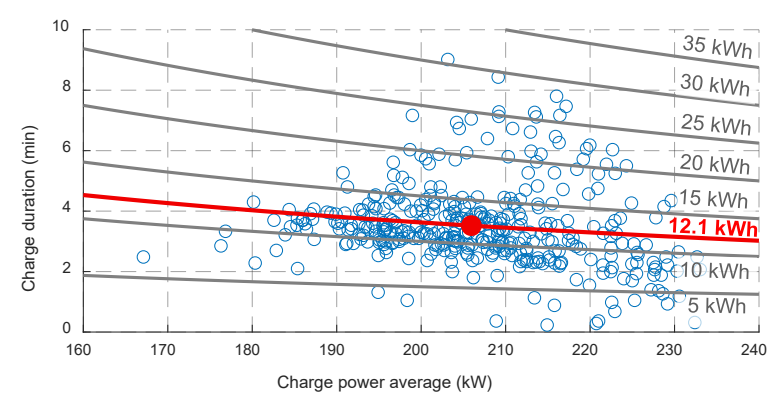

Fig. 4. Power average and duration of the charges. (a) From April 1 to 7 , 2019. (b) From May 13 to 19, 2019. (c) From June 3 to 9, 2019.

week, so it can be expected that the charges throughout the year resemble those made for this study.

TABLE I. CHARGES AVERAGE VALUES

\begin{tabular}{|c|c|c|c|c|c|}
\cline { 2 - 6 } \multicolumn{1}{c|}{} & No. & $\begin{array}{c}\text { Duration } \\
(\text { min })\end{array}$ & $\begin{array}{c}\text { Anticipation } \\
(\text { min })\end{array}$ & $\begin{array}{c}\boldsymbol{P}_{\text {mean }} \\
(\boldsymbol{k W})\end{array}$ & $\begin{array}{c}\boldsymbol{E} \\
(\boldsymbol{k W h})\end{array}$ \\
\hline April, 1 - 7 & $\begin{array}{c}362 \\
(79 \%)\end{array}$ & 3.45 & 3.2 & 203.4 & 11.7 \\
\hline May, 13 - 19 & $\begin{array}{c}285 \\
(62 \%)\end{array}$ & 3.57 & 3.02 & 204.1 & 12.1 \\
\hline June, 3 - 9 & $\begin{array}{c}378 \\
(82 \%)\end{array}$ & 3.52 & 3.92 & 206 & 12.1 \\
\hline Total & $\begin{array}{c}1,025 \\
(74 \%)\end{array}$ & 3.5 & 3.42 & 204.6 & 12 \\
\hline
\end{tabular}

The average value of the power and duration, along with the anticipation are used to make the forecast of the charges. The forecast proposed is a rectangular pulse that takes the value of the average power, which lasts for the average duration obtained in Table I. Regarding the departure time of the bus from the charging station, the moment in which the pulse begins is the sum of the average duration and anticipation of the charges.

\section{ENERGY MANAGEMENT STRATEGIES}

Three energy management strategies are proposed and analyzed in this section in order to reduce the power demanded from the grid in the EV-SCS. Each strategy is explained in more detail below. 


\section{A. Moving Average Strategies}

Since the target variable is the power of the charging point, the moving average strategies make the average value of this parameter. These strategies are interesting because they minimize the peak power. The number of samples $(n)$ on which the moving average is made depends on the moving average time and the sampling period. The longer the moving average time, the larger is the window in which the average value of $P_{C P}$ is made, thereby reducing the influence of the current value. And the shorter the sampling period, the more $P_{C P}$ data is available to perform the moving average.

Two types of moving average strategies are studied. The first one is the simple moving average (SMA) strategy, which only uses previous values of $P_{C P}$ [14]. The second one is the central moving average (CMA) strategy, which also uses future values [15]. Therefore, the CMA strategy requires forecast of the charging point power $\left(P_{C P, f C}\right)$.

In principle, in these strategies the energy balance between the energy demanded at the charging point $\left(E_{C P}\right)$ and the energy of the grid $\left(E_{\text {grid }}\right)$ is maintained, that is, $E_{C P}=E_{\text {grid }}$. However, due to the differences that may exist between the two energies, the ESS would be overcharged or discharged, so a state of charge (SOC) control would have to be introduced.

\section{1) Simple Moving Average Strategy}

The SMA strategy consists of making the average value of $P_{C P}$ from the last value that the moving average achieves $\left(P_{C P-n+1}\right)$ up to the current value $\left(P_{C P_{0}}\right)$. In this way, the SMA power of the charging point $\left(P_{C P, S M A}\right)$ is calculated as:

$$
P_{C P, S M A}=\frac{1}{n} \sum_{i=1}^{n} P_{C P i-n}
$$

Therefore, the power demanded from the grid would be $P_{\text {grid }}=P_{C P, S M A}$, as shown in Fig. 5, while the power difference between the grid and the charging point would be assumed by the ESS.

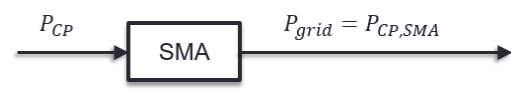

Fig. 5. Structure of the SMA strategy.

In this case, the energy consumption of the grid is equal to the SMA energy of the charging point $\left(E_{C P, S M A}\right)$. As the mean value is made, the energy value does not vary, $E_{\text {grid }}=$ $E_{C P, S M A}=E_{C P}$.

\section{2) Centered Moving Average Strategy}

The CMA strategy requires the prediction of $P_{C P}$, in such a way that it allows the moving average to be carried out with the registered past values and with the predicted future values. By predicting the charging point demand correctly, the size of the ESS to be installed can be reduced compared to the previous strategy.

On the one hand, half of $n$ are taken from the real $P_{C P}$, from the last value that the moving average achieves in half the time $\left(P_{C P-n / 2+1}\right)$ up to the value that is in the current moment $\left(P_{C P_{0}}\right)$. On the other hand, the other half of the samples are the predicted power, from the next instant of time $\left(P_{C P, f c_{1}}\right)$ to the last value that the moving average window reaches $\left(P_{C P, f c_{n / 2}}\right)$. In this way, the centered mean value of the charging point power $\left(P_{C P, C M A}\right)$ is figured out as follows:

$$
P_{C P, C M A}=\frac{1}{n}\left(\sum_{i=1}^{n / 2} P_{C P_{i-n / 2}}+\sum_{i=1}^{n / 2} P_{C P, f c_{i}}\right)
$$

The CMA strategy can be considered a combination of the SMA along with an advanced moving average (AMA) for the $P_{C P, f c}$. Thus, $P_{C P, C M A}$ can also be represented as follows:

$$
P_{C P, C M A}=\frac{P_{C P, S M A}+P_{C P, f C, A M A}}{2}
$$

In this strategy, the CMA energy value of the charging point $\left(E_{C P, C M A}\right)$ not only depends on $E_{C P}$, but also on the predicted energy for the charging point $\left(E_{C P, f C}\right)$. In the event that the forecast differs from what is actually demanded, and that this is not corrected, a difference would be produced between $E_{C P, C M A}$ and $E_{C P}$ based on the following expression:

$$
E_{C P, C M A}=\frac{E_{C P}+E_{C P, f c}}{2}
$$

To correct the error that may occur in the forecast, it is proposed to add half the difference between $P_{C P}$ and $P_{C P, f C}$ to $P_{C P, C M A}$. The energy balance in this case is:

$$
E_{\text {grid }}=\frac{E_{C P}+E_{C P, f c}}{2}+\left(\frac{E_{C P}-E_{C P, f c}}{2}\right)=E_{C P}
$$

The proposed way to add the half of the difference between $P_{C P}$ and $P_{C P, f c}$, is to perform the SMA of this value as shown in the diagram in Fig. 6. It is more likely to guess the energy consumption of a period, instead of an instant. In this way, the influence of the error that may occur in the forecast is decreased.

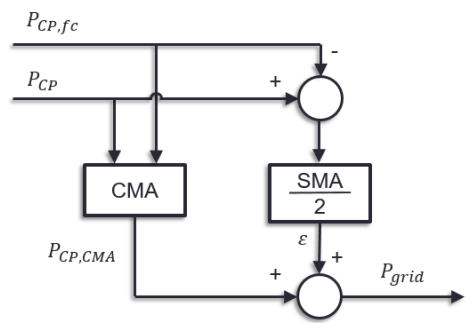

Fig. 6. Structure of the CMA strategy.

\section{B. SOC control strategy}

The SOC control strategy consists of the decision-making tree shown in Fig. 7. The control decision is made depending on whether a bus charge is occurring in the EV-SCS or not. To use this strategy, the SOC of the ESS and the period of time in which the next charge will take place $\left(t_{\text {next charge }}\right)$ to be known.

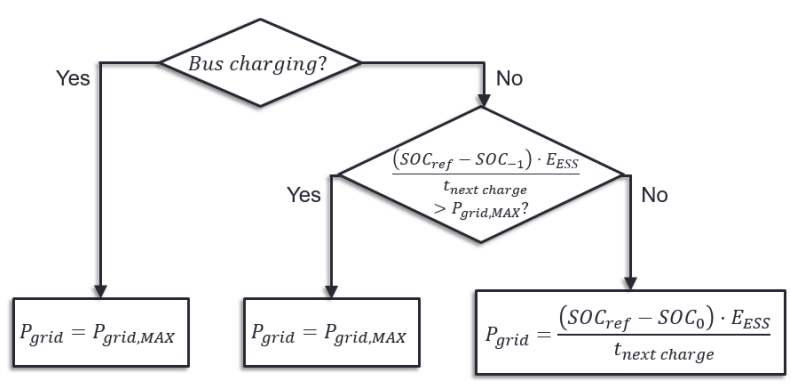

Fig. 7. Decision-tree for the SOC control strategy. 
On the one hand, when no charge is being performed, the $P_{\text {grid }}$ required to charge the ESS is calculated. The calculation of $P_{\text {grid }}$ depends on $t_{\text {next charge }}$, and the SOC current value of the ESS $\left(S O C_{0}\right)$ along with the SOC value in which it is wanted to have the ESS $\left(S O C_{r e f}\right)$ at the moment in which the bus charge.

$$
P_{\text {grid }}=\frac{\left(S O C_{r e f}-S O C_{0}\right) \cdot E_{E S S}}{t_{\text {next charge }}}
$$

To avoid excessive $P_{\text {grid }}$ in the cases when there is not enough time to reach $S O C_{r e f}$, the grid power is limited to a certain maximum value $\left(P_{\text {grid, } M A X}\right)$.

Similarly, when a bus is being charged, the required grid power is $P_{\text {grid,MAX }}$. $P_{\text {grid }}$ with the maximum value implies that $100 \%$ of the grid power that the installation must have is used. In this way, the discharge of the stationary ESS of the EV-SCS is minimized during the buses charge, reducing the required size of the ESS.

\section{SIZING OF THE ESS}

In this section, the sizing of the ESS is carried out based on the simulations of the different strategies proposed in Section IV, using for this the experimental data analyzed in Section III.

In order to obtain the design power based on the size of the ESS, a sweep of the moving average time is carried out in the case of the moving average strategies, and for each moving average time the design power and size of the ESS required are obtained. In the case of the SOC control strategy, a sweep of the maximum grid power allowed in the strategy is performed. Given that this parameter is the design power, a size for the ESS is obtained from each simulation of $P_{\text {grid,MAX }}$.

Fig. 8 shows the power values that would be in the EVSCS with a ESS of $40 \mathrm{kWh}$. The case shown is the operation of the strategies: SMA, CMA with perfect forecast and SOC control. Among the moving average strategies, the variation of $P_{\text {grid }}$ with CMA is lower than the obtained with SMA strategy. In addition, the change in $P_{\text {grid }}$ is less pronounced. Also, in the SMA strategy, higher $P_{\text {grid }}$ values are reached than with the CMA strategy, and the charge and discharge power of the battery is usually greater. In contrast, the SOC control strategy, despite having step changes in the grid demand, $P_{\text {grid }}$ and $P_{E S S}$ are lower, as can be seen in the
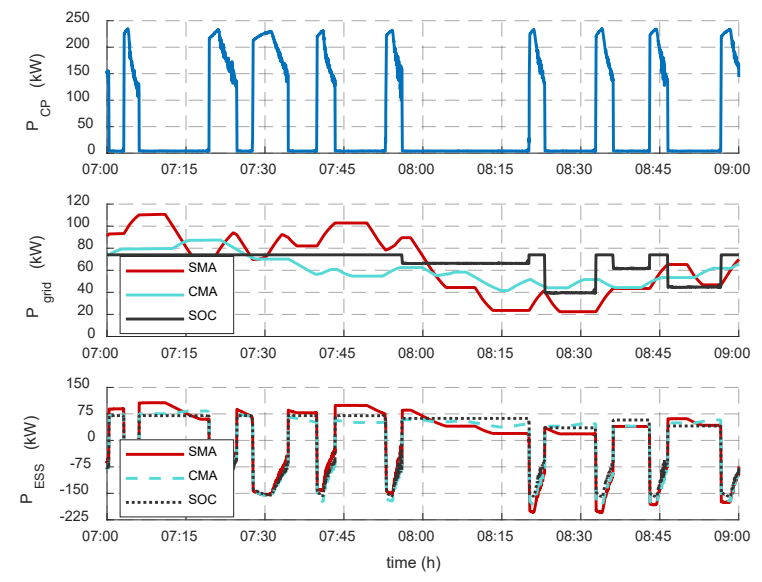

Fig. 8. Power of the EV-SCS with a $40 \mathrm{kWh}$ ESS for the SMA, CMA with perfect forecast and SOC control strategies. demand for $P_{\text {grid }}$ between 7:00 and 7:30, or in the battery discharge that occurs at 8:33.

Fig. 9 shows the maximum grid power for each strategy based on the energy capacity of the ESS. Regarding the CMA strategy, three cases are presented. The first one maintains the same moving average time for the SMA and CMA blocks; in the second one, the time of the SMA block is half of the CMA block; and the last one assumes a perfect forecast. The graph shows that a larger ESS allows reduction of the maximum power demanded from the grid with any of the proposed strategies.

Regarding the CMA strategies that do not make a perfect forecast, when using moving average lower than 12 minutes (bus frequency), the error in the forecast has the opposite effect to the one desired to reduce $P_{\text {grid }}$. Therefore, this strategy is not suitable for batteries smaller than $20 \mathrm{kWh}$.

Comparing the perfect-forecast CMA strategy with those with real forecast, it is observed that the first one offers better results. It is shown the potential to improve the proposed forecast. If the forecast cannot be improved, the most suitable option among the moving averages strategies is the SMA strategy, with which the same results are obtained as with the CMA strategy without perfect forecast. In addition to offering the same results, the required data and computation power are lower, and it turns out to be an easier strategy to implement.

Compared to moving average strategies, there is the SOC control strategy. This one reduces the maximum grid power more than the other strategies. Regarding the CMA strategy with perfect forecast, it is possible to reduce the surrounding power by $20 \%$ more. Meanwhile, compared to the rest of the moving average strategies, $P_{\text {grid,MAX }}$ is reduced by up to $40 \%$ with the same size of ESS as in the other strategies. Without a ESS, the maximum power reached is $250 \mathrm{~kW}$. When installing a $40 \mathrm{kWh}$ ESS, this value with the SOC control strategy is reduced to $74 \mathrm{~kW}$, that is, a reduction greater than $70 \%$. For the CMA strategy with perfect forecast, $P_{\text {grid,MAX }}$ is $96 \mathrm{~kW}$, and for the other strategies it reaches a value of $140 \mathrm{~kW}$, representing $38 \%$ and $56 \%$ of $250 \mathrm{~kW}$ respectively.

In the case of Spain, in the term of the contracted power in the electricity rate for powers greater than $15 \mathrm{~kW}$, the maximum recorded value is not taken, but the highest value of the fifteen-minute average of the power registered during the month, therefore, to perform the analysis on the reduction in contracted power that is achieved by introducing a ESS in the

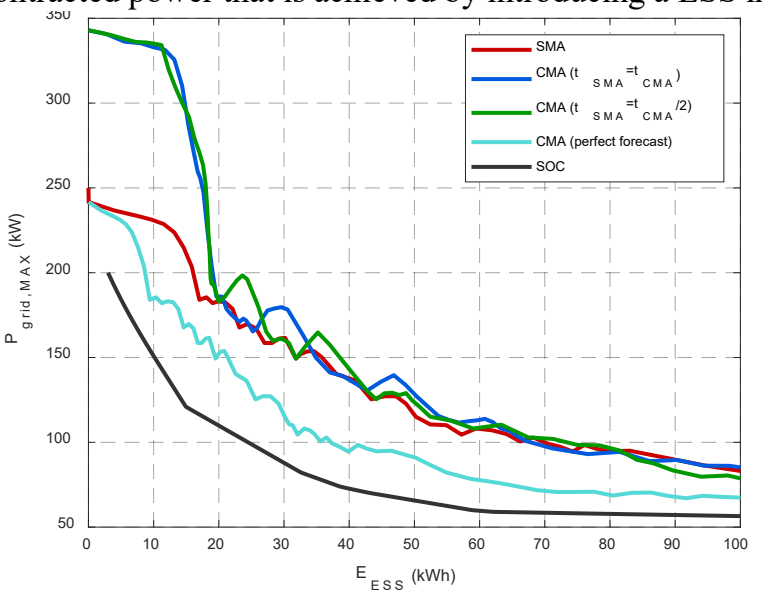

Fig. 9. Comparison of the strategies for the maximum grid power needed depending on the ESS energy. 
EV-SCS, the fifteen-minute average of the power is used, which is shown in Fig. 10 for each of the strategies. Since the fifteen-minute average is performed, instead of the instantaneous value, the power value is lower or equal to those represented in Fig. 10. For the SOC control strategy, the curve is practically the same as the curve in Fig .10, which means that for at least 15 minutes the grid power is equal to $P_{\text {grid,MAX }}$. While, for moving average strategies, the curves are modified to a greater extent, which means that the maximum value is taken at specific moments.

Without ESS, the contracted power required is $180 \mathrm{~kW}$, and with a $40 \mathrm{kWh}$ ESS, all strategies reduce this value. Moving averages strategies reduce the value to $122 \mathrm{~kW}$, which is $68 \%$ of $180 \mathrm{~kW}$. In the case of the CMA strategy with perfect forecast, the power drops to $94 \mathrm{~kW}$, a reduction of $52 \%$. And just as for the design power of the grid, the SOC strategy is the one that offers the best results, with a contracted power of $72 \mathrm{~kW}$ for this strategy, it means the $40 \%$ of $180 \mathrm{~kW}$.

\section{Conclusions}

The increase in electric vehicle charging stations rises the interest on the sizing of these facilities to favor their installation. This contribution studies various strategies to improve the sizing and management of such installation. According to the results obtained in section IV, a $40 \mathrm{kWh}$ battery, allows for a reduction of $70 \%$ in the connection power to the grid. For the same size of the ESS, the SOC control strategy is the one that gest reduce the power grid demand the most. It is true that with this strategy the power peaks are reduced, but they do not disappear because of how the strategy works and, depending on the power peaks, the destabilization of weak distribution grids can be produced [17]. In such cases, the power peaks of the SOC control strategy could be solved by implementing ramp control. By contrast, moving average strategies avoid the power peaks in the demand and automatically the demanded power change with ramps, being more favorable for grid stability in weak distribution. These ramps depend on the moving average time and the power difference between the new values introduced in the moving average and the last values took off from the moving average. The ramps are not controlled, but with the demanded power expected in the charging point and the moving average time chose, the ramps gradient can be calculated. For the moving average strategies, due to the fact that may exist a difference between the energy of the charging point and the energy of the grid, a SOC control should be introduced in the structure. In

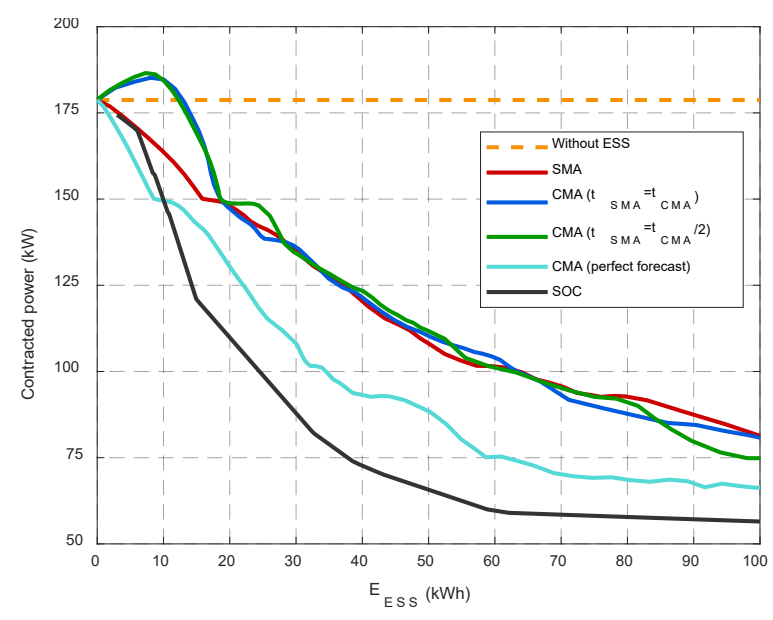

Fig. 10. Comparison of the strategies for the contracted grid power needed depending on the ESS energy. turn, the aging of the energy storage system is a point to take into account in order to preserve it from premature degradation.

\section{REFERENCES}

[1] I. Burch and J. Gilchrist, "Survey of Global Activity to Phase Out Internal Combustion Engine Vehicles," no. September, p. 14, 2018.

[2] L. Ingeborgrud and M. Ryghaug, "The role of practical, cognitive and symbolic factors in the successful implementation of battery electric vehicles in Norway," Transp. Res. Part A Policy Pract., vol. 130, no. October, pp. 507-516, 2019.

[3] C. McKerracher et al., "Electric Vehicle Outlook 2019," BloombergNEF, 2019. https://about.bnef.com/electric-vehicleoutlook/ (accessed Feb. 10, 2020).

[4] Energeia, “Australian Electric Vehicle Market Study,” Energeia, pp. 1103, 2018.

[5] T. Placke, R. Kloepsch, S. Dühnen, and M. Winter, "Lithium ion, lithium metal, and alternative rechargeable battery technologies: the odyssey for high energy density," J. Solid State Electrochem., vol. 21, no. 7, pp. 1939-1964, 2017.

[6] J. W. Brennan and T. E. Barder, "Battery Electric Vehicles vs. Internal Combustion Engine Vehicles," 2016.

[7] N. Lutsey and M. Nicholas, "Update on electric vehicle costs in the United States through 2030," Int. Counc. Clean Transp., no. June, pp. $1-12,2019$.

[8] A. Gupta and A. Semwal, "EV Charging Infrastructure Market Size By Current (AC \{Level 1, Level 2\}, DC), By Charging Site (Public, Private) Industry Analysis Report, Regional Outlook, Price Trends, Growth Potential, Competitive Market Share \& Forecast, 2019 2025," Glob. Mark. Insights, p. 360, 2019.

[9] A. Nightingale, "Forget Tesla, It's China's E-Buses That Are Denting Oil Demand," Bloomberg, 2019. https://www.bloomberg.com/news/articles/2019-03-19/forget-tesla-its-china-s-e-buses-that-are-denting-oil-demand (accessed Feb. 10, 2020).

[10] A. M. Othman, H. A. Gabbar, F. Pino, and M. Repetto, "Optimal electrical fast charging stations by enhanced descent gradient and Voronoi diagram," Comput. Electr. Eng., vol. 83, p. 106574, 2020.

[11] D. A. Savio, V. A. Juliet, B. Chokkalingam, S. Padmanaban, J. B. Holm-Nielsen, and F. Blaabjerg, "Photovoltaic integrated hybrid microgrid structured electric vehicle charging station and its energy management approach," Energies, vol. 12, no. 1, 2019.

[12] "Shell ultrafast EV charging service to incorporate Alfen energy storage," Alfen, 2020. https://alfen.com/en/news/shell-ultrafast-evcharging-service-incorporate-alfen-energy-storage (accessed Mar. 18, 2020).

[13] J. P. Torreglosa, P. García-Triviño, L. M. Fernández-Ramirez, and F. Jurado, "Decentralized energy management strategy based on predictive controllers for a medium voltage direct current photovoltaic electric vehicle charging station," Energy Convers. Manag., vol. 108, pp. 1-13, 2016.

[14] J. Pascual, P. Sanchis, and L. Marroyo, "Implementation and control of a residential electrothermal microgrid based on renewable energies, a hybrid storage system and demand side management," Energies, vol. 7, no. 1, pp. 210-237, 2014.

[15] J. Pascual, J. Barricarte, P. Sanchis, and L. Marroyo, "Energy management strategy for a renewable-based residential microgrid with generation and demand forecasting," Appl. Energy, vol. 158, pp. 12$25,2015$.

[16] A. Soto, A. Berrueta, P. Sanchis, and A. Ursua, "Analysis of the main battery characterization techniques and experimental comparison of commercial 18650 Li-ion cells," in Proceedings - 2019 IEEE International Conference on Environment and Electrical Engineering and 2019 IEEE Industrial and Commercial Power Systems Europe, EEEIC/I and CPS Europe 2019, 2019, pp. 1-6.

[17] G. Hoogsteen, A. Molderink, J. L. Hurink, G. J. M. Smit, F. Schuring, and B. Kootstra, "Impact of peak electricity demand in distribution grids: A stress test," 2015 IEEE Eindhoven PowerTech, PowerTech 2015, pp. 1-6, 2015. 